\title{
A spatio-temporal analysis of BSE cases born before and after the reinforced feed ban in France
}

\author{
Christian DUCROT ${ }^{a *}$, David ABRIAL $^{\mathrm{a}}$, Didier CALAVAS ${ }^{\mathrm{b}}$, \\ Tim CARPENTER ${ }^{\mathrm{C}}$
}

\author{
a Unité d'Epidémiologie Animale, INRA Theix, 63122 Saint-Genès-Champanelle, France \\ b Unité Epidémiologie, AFSSA Lyon, 31 avenue T. Garnier, 69364 Lyon Cedex 07, France \\ ${ }^{c}$ Center for Animal Disease Modeling and Surveillance (CADMS), School of Veterinary Medicine, \\ University of California, Davis, CA 95616, USA
}

(Received 7 December 2004; accepted 12 May 2005)

\begin{abstract}
A spatio-temporal analysis was carried out to see how the risk distribution of bovine spongiform encephalopathy (BSE) in France changed depending on the period of birth. The data concerned the 539 BSE cases born in France after the ban (BAB) of meat and bone meal (MBM) in 1990 and detected between July 1, 2001 and December 31,2003, when the surveillance of BSE was comprehensive. Seventy-two of these cases were born after the reinforced (second) ban (BASB) in 1996, which involved the removal of BSE-risk materials and cadavers from the processing of MBM. The Ederer-Myers-Mantel (EMM) time and space cluster test was applied, after classifying the cases by trimester and region of birth, BAB or BASB status, and dairy or beef status. Then disease mapping was performed for four successive birth periods, three for the BAB cases (January 1991 through June 1994, July 1994 through June 1995, July 1995 through June 1996), and one for the BASB (July 1996 through October 1998). It was elaborated with the Bayesian graphical modelling methods and based on a Poisson distribution with spatial smoothing. The parameters were estimated by a Markov Chain Monte Carlo (MCMC) simulation method. The main finding was that the areas with the highest risk of BSE changed largely from one birth period to another; from the west, it reached the east of France for birth cohort 1994-1995 and the southwest for birth cohort 1995-1996. The EMM test identified a peak risk in this region both for dairy and beef cattle in the fall 1995. The spatial distribution of the risk for the BASB cases matched the spatial pattern of risk for the preceding $\mathrm{BAB}$ birth cohort quite well; this was in favour of a common origin of the infection of the $\mathrm{BAB}$ and $\mathrm{BASB}$ cases, despite the complementary control measures.
\end{abstract}

BSE / bovine / spatio-temporal / disease mapping / Ederer-Myers-Mantel

\section{INTRODUCTION}

A meat and bone meal (MBM) ban for cattle was implemented in France in $1990^{1}$ in order to reduce the incidence of bovine spongiform encephalopathy (BSE). In spite of this action, BSE cases continued to be identified in cattle born after the ban (BAB). This resulted in an even more stringent feed ban being implemented in June $1996^{1}$. In order to eliminate cross contamination of ruminant feed with feed containing MBM

\footnotetext{
* Corresponding author: ducrot@ clermont.inra.fr

1 http://www.agriculture.gouv.fr/esbinfo/esbinfo.htm [consulted 07 March 2005].
} 
fed to non-bovines, cadavers and specified risk material (SRM) were removed from the processing of MBM used in pig and poultry feed. As of October 15, 2004, 96 BSE cases had been identified in animals born in France after the second ban (BASB).

As of October 6, 2003, 59 BSE cases in Great Britain were animals born after the total (real) ban (BARB) on using mammalian meat and bone meal (MMBM) in any farm animal feed ${ }^{2}$ (Wilesmith et al. ${ }^{3}$ ). The British BARB cases cannot be compared to the French BASB cases, since the control measures were different; however, both were infected when an MBM ban was in force and alternative sources of infection were active. It is believed that BARB BSEcases result from the consumption of contaminated imported feed [32]. In France, hypotheses on the origin of the BASB BSEcases were formulated by an expert panel in 1998 [3]. They concluded BASB BSEcases were likely the result of an unsuccessful MBM ban and cross contamination of ruminant feed with feed containing MBM fed to poultry and pigs. More recently, a case study carried out in 2004 on 34 of these cases showed that maternal transmission can be ruled out for $82 \%$ (28) of the cases, and that $97 \%$ (33) of case farms used purchased proprietary concentrates but only $56 \%$ (19) purchased milk replacer [18].

Disease mapping has been performed on the distribution of the French BSE cases detected after June 2001, when a comprehensive surveillance programme (including clinical surveillance and screening tests on fallen stock and cattle at the abattoir) was in place [1]. It showed the spatial heterogeneity of the BSE risk but did not search for

\footnotetext{
2 Statutory Instrument 1996 No. 2007, The Bovine Spongiform Encephalopathy Order 1996 , coming into force the 1st August 1996, http://www. hmso.gov.uk/si/si1996/Uksi [consulted 07 March 2005].

3 Wilesmith J.W., Stevenson M.A., Morris R.S., Ryan J.B.M., Arnold M., Prince M., An epidemiological update of cases of BSE born after 31 July 1996 in Great Britain, 2003, Report, 25 p.
}

temporal variations of the disease mapping. A more detailed exploration of the spatiotemporal distribution of $\mathrm{BAB}$ and BASB BSE-cases might provide new insights into the origin of those BASB cases. The assumption is that the spatial distribution of BASB BSE-cases is dependent on the source of infection. As such, we would expect the distribution to be random if there was a non-specific source of BSE contamination, as with the sporadic CreutzfeldtJakob disease, or clustered in specific areas. If the latter was true, the BASB BSE clusters could be located where the epidemic was located in France five to seven years ago, supporting the hypothesis of either an environmental or maternal exposure. An alternate location might be in the same regions as for the $\mathrm{BAB} \mathrm{BSE}$-cases born in the previous period; this would support a common origin for the $\mathrm{BAB}$ and $\mathrm{BASB}$ BSE-cases. The purpose of this research was to explore the spatial and spatio-temporal distribution of the BSE cases over time in an attempt to better understand and hasten the eradication of this disease.

\section{MATERIALS AND METHODS}

\subsection{Data}

Epidemiologic data on BSE were provided by the "Agence Française de Sécurité Sanitaire des Aliments" (AFSSA), in charge of BSE monitoring. The analysis was restricted to cases detected between July 1 , 2001 and December 31, 2003 in order to get precise and comparable data on BSE incidence; during this period, the detection of BSE was based both on the Mandatory Reporting System and the comprehensive active surveillance programme, which uses rapid tests that passed the European Union validation [25] and is carried out on all slaughtered and fallen cattle at least 2 years old $[6,7]$. BSE cases examined here were confirmed at the national reference laboratory of AFSSA with Western blot or immunohistochemistry [6]. During the study 


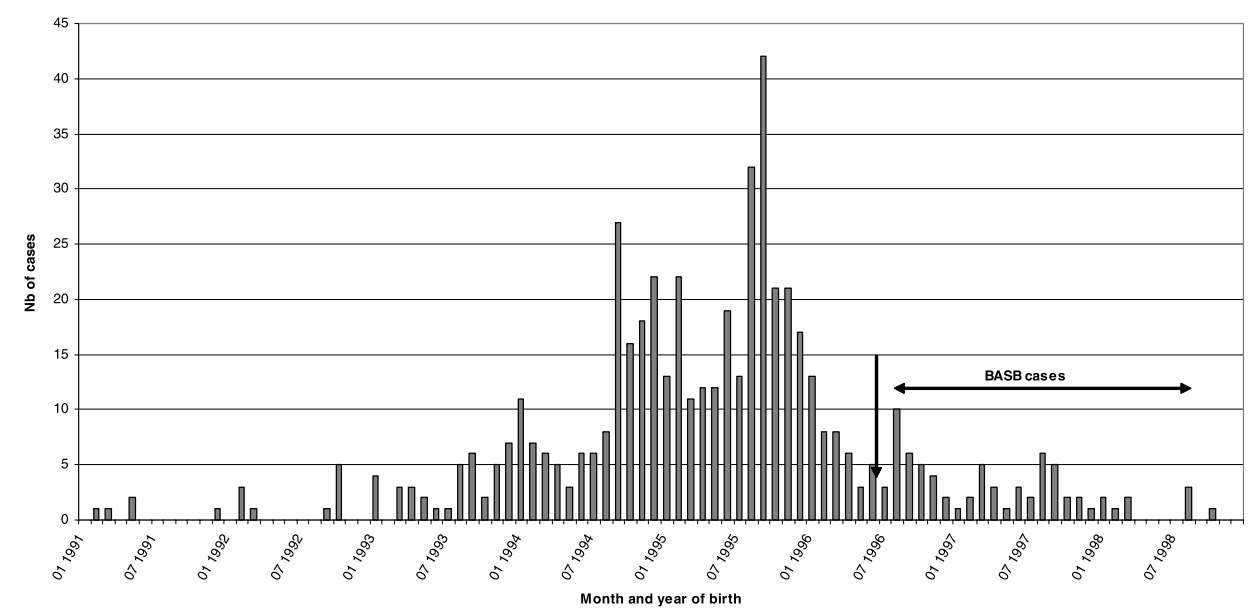

Figure 1. Temporal distribution of the 539 BAB and BASB BSE cases detected from July 2001 through December 2003 in France, depending on the month and year of birth.

period, 539 BSE cases were detected in France, 441 in dairy and 98 in beef cattle. Four-hundred and sixty-seven BSE cases were BAB and $72 \mathrm{BASB}$. The dates of birth are shown in Figure 1. Most (503/539) of the BAB (438/467) and BASB (65/72) cases were born from July 1993 through December 1997.

The geographic location of a BSE case was defined as the location of the "commune" (the smallest French administrative unit $-150 \mathrm{~km}^{2}$ on average) of the farm where the case was raised between the sixth and twelfth months after birth. This period corresponds to the highest risk of infection, based on modelling results [12, 30]. BSE incidence varies according to the production type (dairy versus beef cattle) [13, 23, 31]. These types were taken into account in the standardisation of BSE cases for the disease mapping: the expected count of BSE cases under the null hypothesis of homogenous spatial distribution. We defined the production type according to the breed of the case, if known, or the production type of the farm when the breed was not recorded or when the case was of a mixed breed.
The population at risk was defined as the number of cows at the "canton" level (French administrative unit including five "communes" on average; France is divided into 3705 cantons). Data were obtained from the Agricultural Census 2000 edited by the Statistics Office of the Ministry of Agriculture and Fisheries (Agreste, 251 rue de Vaugirard, 75732 Paris, France). The geographical data on the cantons' perimeters and the communes' centroids were provided by the GEOFLA ${ }^{\circledR}$ "France Métropolitaine" (IGN ${ }^{\circledR}$ Paris, version 6, 2002).

\subsection{Statistical methods}

The statistical analysis was carried out in two steps.

\subsubsection{Analysis of clusters in space and time}

The first step of the analysis was based on the Ederer-Myers-Mantel (EMM) test $[14,20]$. The null hypothesis was that the number of cases identified over time did not differ between regions. The BSE cases were classified by region of origin at birth. The 
French metropolitan territory is divided into 22 regions (administrative units). Because only 28 of the 467 BAB cases were born between 1991 and June 1993, the analysis of the BAB cases was limited to cases born between July 1993 and June 1996, and the time periods were defined as the trimester of birth.

The EMM test [14] aimed at identifying the presence of clusters in time and space, simultaneously. It is different from a timespace clustering because it detects a temporal clustering of events in specific locations, simultaneously, without regards to the proximity of locations to one another [8]. It was used to check if the time distribution of the trimester of birth varied between regions. The EMM technique works as follows: the total number of cases is grouped by time period of occurrence, e.g. month, season and by spatial unit, e.g. building, census tract, city, ZIP code, administrative unit, county, or state. The maximum number of cases $\left(m_{1}\right)$ in any time period $\left(s_{\mathrm{i}}\right)$ is then compared with the total number of cases $(r)$, observed in that location. Assuming the cases follow a Poisson distribution, they are expected to occur in the time periods, according to a multinomial distribution. Using this technique, the summation of $m_{1}$ $\left(\Sigma m_{1}\right)$ across locations is compared with the summation of the expected maximum number $\Sigma\left(E\left(m_{1}\right)\right)$, given the total number of cases reported and the time periods examined.

The EMM-test statistic is based on the probability of detecting a disease cluster in a single time period, conditional on the number of cases observed over the entire period. The null hypothesis is that the cases were randomly and independently distributed, with a probability of $1 / s$, where $s$ is the number of time periods. The rejection of this hypothesis supports the assumption that temporal clustering exists in at least one of the spatial units.

A comparison of variations from the expected is performed by a one-tailed chi- square test with a single degree of freedom and continuity correction as

$$
\chi^{2}=\frac{\left[\sum m_{i}-E\left(\sum m_{i}\right)-0.5\right]^{1 / 2}}{\sum V\left(m_{i}\right)},
$$

where, $m_{i}=$ the maximum number of cases observed in a single time period in area $i$, $\sum m_{i}=$ the sum of the maximum number of cases observed in a single time period for each of the areas, 0.5 is the continuity correction factor, used when the absolute value term calculated in equation 4 is greater than 0.5 , and $V\left(m_{i}\right)=$ the variance of the area maxima.

The expected sum of $m_{i}$ is based on the method for determining the probability of distributing $r$ balls (total cases) in $n$ cells (time periods) [15]. Calculations for the expected maxima and variances for various combinations of $r$ and $n$ have been presented elsewhere [14, 20, 27, 28].

Two different EMM analyses were carried out on the $\mathrm{BAB}$ cases, separating dairy from beef cattle cases, because the seasonal pattern of birth for dairy and beef cows is substantially different. The method was then applied to the BASB cases, those born after June 1996, without separating dairy and beef cases due to the limited number of cases. The analysis was performed with the SSTATV470b spatial statistical software (University of California, Davis, CA, USA).

The EMM test can be influenced by the annual trend of the infection, and also by the seasonal trend of the period of birth of cattle. So we again computed the EMM test, by pooling data first on the year of birth ( 3 time periods corresponding to the 3 years), and second on the season of birth (4 time periods corresponding to the seasons, whatever the year of birth).

\subsubsection{Disease mapping}

The second step was a disease mapping model based on a Poisson distribution. The null hypothesis was that the cases born in a 
certain time period were randomly distributed in France. Four different analyses were built to examine the spatial distribution of the BSE risk for different successive birth periods. The first three corresponded to the BAB cases, with the following birth periods: January 1991 through June 1994; July 1994 through June 1995, and July 1995 through June 1996. The last birth period corresponded to the BASB cases and ranged from July 1996 through October 1998.

The disease mapping was carried out with the same methodology as that explained in detail in Abrial et al. [1]. To summarise, the geographical units were defined as 1264 contiguous hexagons of $23 \mathrm{~km}$ width and $450 \mathrm{~km}^{2}$ area, covering Metropolitan France. The BSE cases, geographically referenced by the centroid of the "commune", were aggregated at the hexagon level by a simple count of BSE "points" for which the "commune" fell into the hexagon. The population of the female adult cattle, available at the "canton" level, was estimated at the hexagon level by aggregating the population of the different "cantons" of concern, weighted by the percentage of area overlapping between each "canton" and the hexagon. All these spatial operations used the union function of the Geographical Information System (ArcView GIS, ESRI Inc., Redlands, CA, USA).

The count of the observed BSE cases $y_{i}$ in the hexagon $i$ was compared to the expected number $e_{i}$ in the same hexagon with the hypothesis that the BSE risk was homogeneously distributed on the spatial scale. This ratio provided a Standardised Incidence Ratio $S I R_{i}$, which represented an increase or decrease of the risk of contamination in the hexagon compared to the overall standard risk evaluated for all France. The expected numbers of BSE cases were assessed by taking into account the proportion of dairy and beef population at risk.

$$
\begin{aligned}
e_{i} & =p_{\text {dairy }} . D_{A I R} Y_{i}+p_{\text {beef }} \cdot B E E F_{i} \\
i & =1 \ldots 1264 .
\end{aligned}
$$

In this equality, $p_{\text {dairy }}$ and $p_{\text {beef }}$ are respectively the probability of a dairy and a beef animal being contaminated. They are assessed by the ratio count of BSE cases in the bovine population. DAIRY $Y_{i}$ and $B E E F_{i}$ are the numbers of bovines in the hexagon $i$.

We assumed that the observed number of BSE cases followed a Poisson distribution (rare event and large number of hexagons) with parameter $\lambda_{i}$.

$$
\begin{gathered}
y_{i} \sim \text { Poisson }\left(\lambda_{i}\right) \\
\log \left(\lambda_{i}\right)=\log \left(e_{i}\right)+u_{i}+v_{i} \\
\operatorname{SIR}_{i}=\frac{\lambda_{i}}{e_{i}}=\exp \left(u_{i}+v_{i}\right) \\
i=1 \ldots 1264 .
\end{gathered}
$$

Within the methodology of the disease mapping with a hierarchical Bayesian approach, the priors on the spatial components $u_{i}$ and $v_{i}$ were expressed as the smoothing of the raw SIR [4]. The component $u_{i}$ took into account the spatial dependence between the hexagons using a notion of "adjacency" between the hexagons. This prior is based on an intrinsic conditional autoregressive model [10]:

$$
u_{i} \sim N\left(\mu_{\partial i}^{u}, \kappa_{i}\right)
$$

$\mu^{u}$ is the mean of the spatial components in the set $\partial i$ of the hexagons adjacent to hexagon $i$ ("adjacency") and $\kappa_{i}$ is the variance inversely weighted by the number of neighbours of hexagon $i$. The $v_{i}$ were the random effects without spatial structure, a Gaussian "white noise" of mean 0 and variance $\tau$.

$$
v_{i} \sim N(0, \tau)
$$

Parameter estimation was carried out with the WinBUGS package version 1.4 (http://www.mrc-bsu.cam.ac.uk/bugs/welcome.shtml). The Monte Carlo Markov Chain (MCMC) method used by WinBUGS is the Gibbs sampling [26]. The analysis provided a Markov chain from which a sufficiently long burn-in of samples (size 50000 cycles) was discarded from calculations, followed by an effective and usable chain (size 100000 cycles). The parameters 


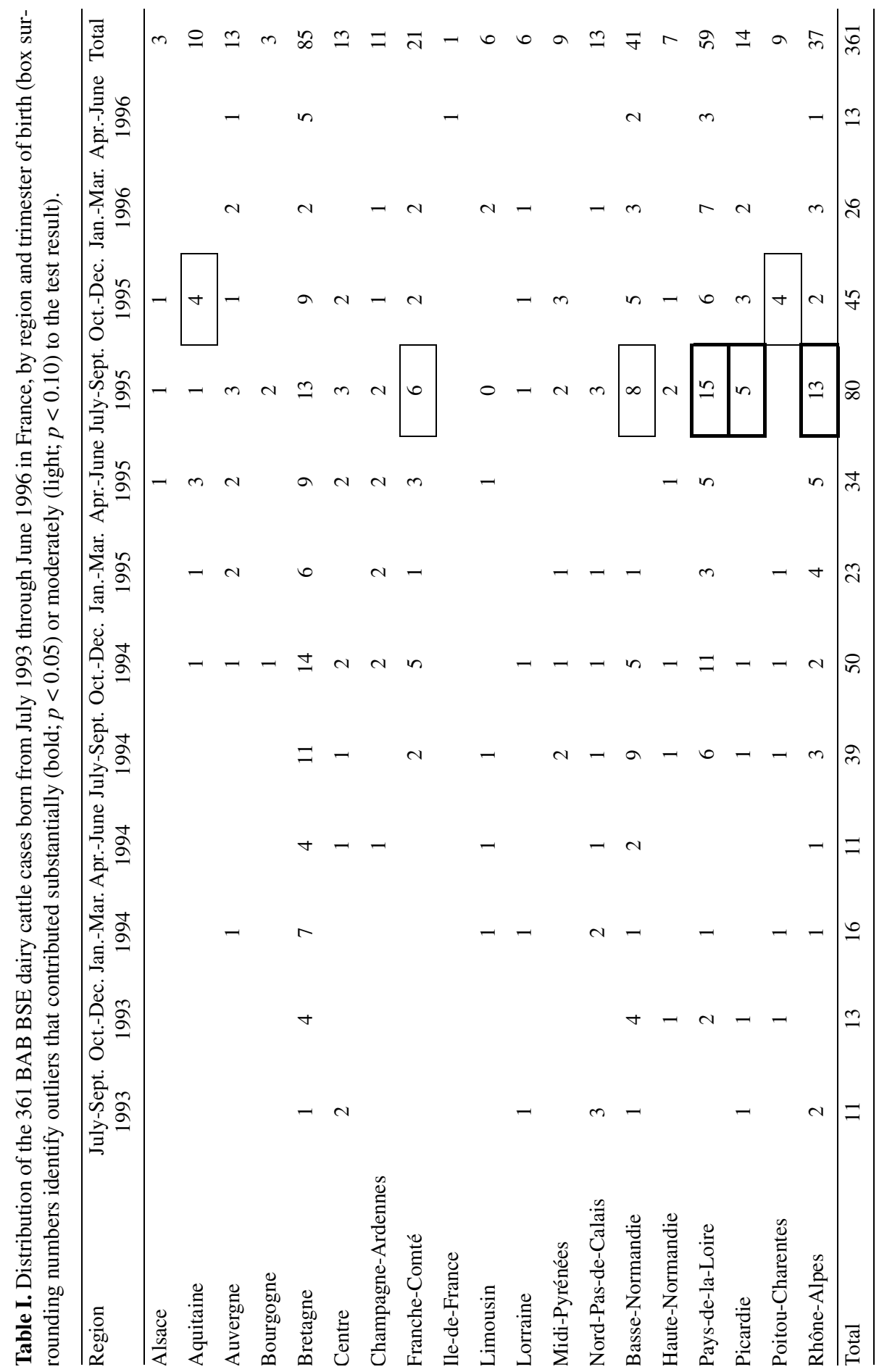




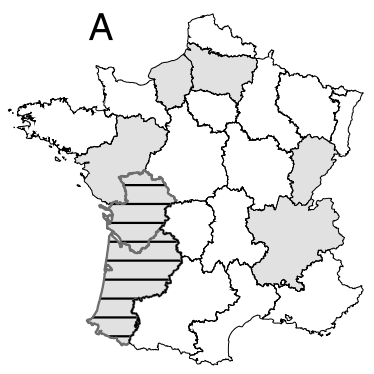

BAB cases-Dairy

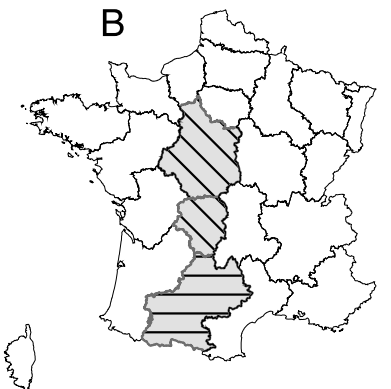

BAB cases-Beef

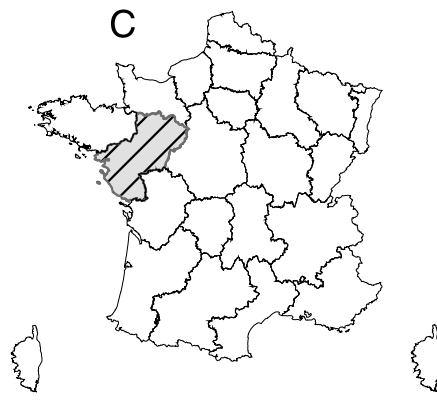

BARB cases (Dairy \& Beef)

Period of birth $\Delta \backslash$ Jan-Mar $95 \square$ July-Sept $95 \square$ Oct-Dec $95 \square /$ July-Sept 97

Figure 2. Summary of the results of Ederer-Meyer-Mantel (EMM) tests of the 511 BAB and BASB BSE cases detected from July 2003 through December 2003 in France, depending on the month and year of birth; areas and birth periods that contribute substantially to the test result.

of interest were estimated from the sampling of 20000 cycles from the chain; only 1 cycle of 5 was used to avoid the autocorrelation between successive cycles. We assumed that the hyperparameter of the variances followed a Gamma prior with shape and scale parameter both equal to 0.01 as suggested by Browne [5]. The stability of the chains was checked with the Heidelberger-Welch convergence diagnostic [17].

In order to identify the hexagons with an SIR significantly higher than 1 and test if the variance of the spatial component was significantly higher than 0 , we computed a $99 \%$ prediction interval based on the $0.5 \%$ and $99.5 \%$ quantiles from the Gibbs sampling of 20000 cycles $[21,22]$. The variability of the spatial component is a measure of the spatial heterogeneity, which enables the evaluation of the spatial distribution of BSE cases.

\section{RESULTS}

\subsection{Analysis of clusters in space and time}

Concerning the BAB BSE cases born from July 1993 through June 1996, the distribution of the cases depending on the region and trimester of birth is shown in Tables I and II for dairy and beef cattle, respectively. The regions with dairy and beef cases represented $84 \%$ and $86 \%$ of the dairy and beef cattle population, respectively. Concerning dairy cattle, the results of the EMM test were highly significant $(p<0.0001)$; three regions contributed significantly $(p<0.05)$ and four moderately $(p<0.10)$ to the chi-square statistic (Tab. I). The peak of cases by trimester of birth varied between regions. More precisely, two contiguous regions of the southwest of France (Aquitaine and PoitouCharentes) had a peak of cases born in the trimester "October-December 1995", whereas it was the previous one (July-September 1995) for five other regions (Fig. 2). Concerning beef cattle, the results of the EMM test were also highly significant $(p=0.0007)$. Three regions contributed significantly $(p<0.05)$ to the chi-square statistic (Tab. II). Interestingly, the region Midi-Pyrénées, in the southwest of France, had a peak of cases for beef cattle born during the same trimester as did BSE dairy cases in contiguous regions (OctoberDecember 1995), whereas the peak was at a different period for the other regions (January-March 1995), three trimesters earlier 


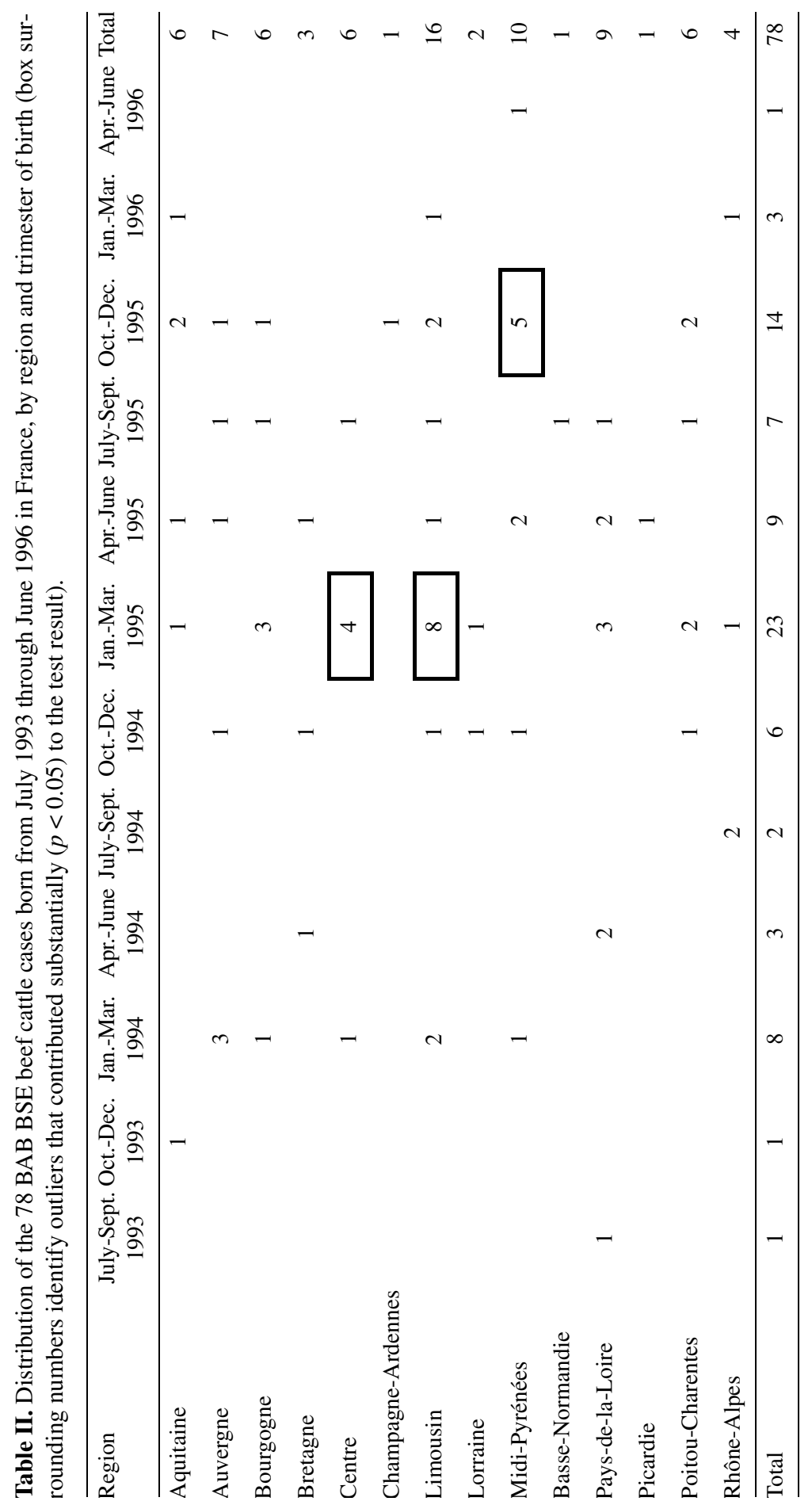


(Fig. 2). The EMM tests performed by pooling data first on the year of birth (3 time periods corresponding to the years), and second on the season of birth (4 time periods corresponding to the seasons), were both significant $(p<0.05)$ for dairy cattle (data not shown). This supports the results from the overall test on the 12 trimester periods that was significant because of both the annual and seasonal trends of the birth date of the BAB BSE cases.

The regions with BASB BSE cases, including dairy and beef, represented $95 \%$ of the French cattle population. The test for the BASB cases was applied (Tab. III) to the overall data set, without distinguishing dairy and beef cattle, because of the very limited number of cases for this time period (only 11 beef cases). The time periods were defined as ten trimesters of birth, starting in July 1996 and ending in December 1998. Only 16 of the 22 French regions had BASB cases. The results of the EMM test were significant $(p=0.05)$. The region Pays-de-laLoire contributed statistically $(p<0.05)$ to the chi-square statistic (Tab. III), during the birth period July through September 1997 (Fig. 2).

\subsection{Disease mapping}

The distribution of the BSE cases in the four birth periods was the following; 90 BAB cases born from January 1991 through June 1994, 189 from July 1994 through June 1995, 188 from July 1995 through June 1996, and the 72 BASB cases born from June 1996 through October 1998. The variance of the spatial component was significantly $(p<0.01)$ greater than zero for the four case categories: BAB cases born in the birth periods January 1991-June 1994 (99\% confidence interval: [1.98-2.08]), July 1994 through June 1995 (0.97-1.07), July 1995 through June 1996 (0.95-1.08) and for the BASB cases born after June 1996 (1.87-2.32). This indicated that there was a significant spatial heterogeneity of the BSE risk for each of the birth periods including those of the BASB cases. For each of these four birth periods, the spatial pattern of BSE cases in France could not be explained by chance alone.

The map of the BSE risk per birth cohort is shown in Figure 3 and the hexagons with a statistically significant higher risk in Figure 4. These maps show that the areas with the highest BSE risk were different for each birth cohort. Located in Brittany, for cohorts born before July 1994, the highest BSE risk areas moved to the center and eastern parts of France for the cohort born from July 1994 through June 1995. Then two new areas were at the highest risk for the cohort born from July 1995 through June 1996, Pays-de-la-Loire and southwest of France. Finally, the map of the BASB cases, born after June 1996 shows the highest risk in the Pays-de-la-Loire region.

\section{DISCUSSION}

The results in this study may have been affected by reporting bias. Specifically, only cases detected after June 2001 were analysed in the study. The reason was that the surveillance was not comprehensive before and biased both spatially and with respect to the production type of the cows $[11,13]$. There are two consequences to this limited analysis. First, given the distribution and the average age at clinical onset that is around 6 years, most of the cases considered in the analysis were born from 1993 through 1997 [9]. Second, all cases originating from these birth cohorts were not included in the analysis, since some were diseased before 2001, especially for the oldest birth cohorts such as those of 1993. Similarly, cases may have developed from the youngest birth cohorts after the conclusion of data collection for this study. In order to handle that bias, the assumption was made that the age on BSE diagnosis was independent of geographic location of either the birth or diagnosis. Accordingly, we assumed that the geographical distribution of BSE cases among a given birth cohort and 


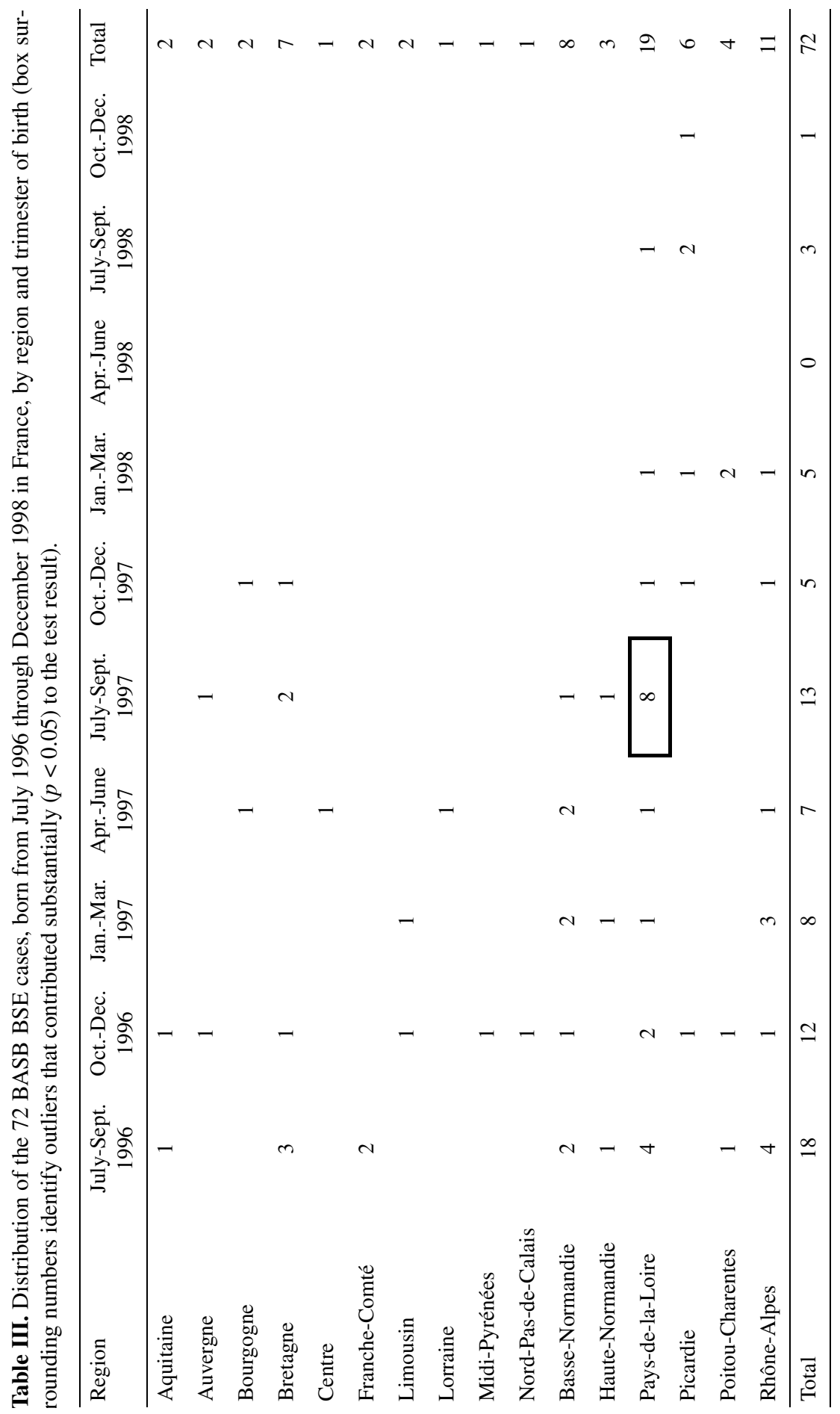



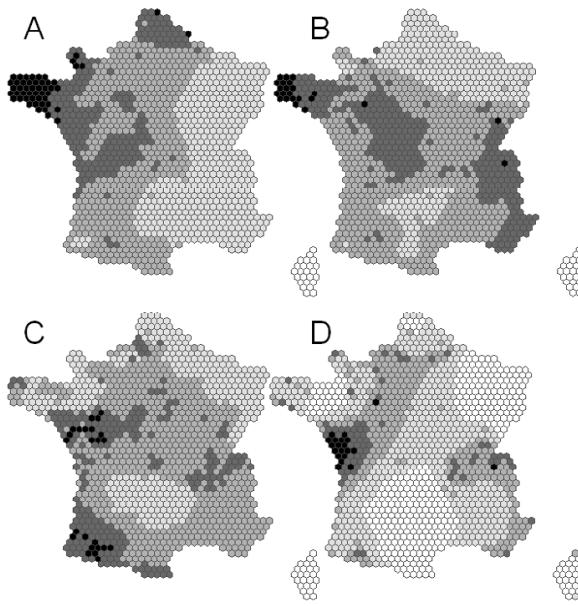

Figure 3. Disease mapping of the standardised incidence ratio (SIR) of the BSE risk for the four different periods of birth: (A) January 1991 through June 1994 (90 cases), (B) July 1994 through June 1995 (189 cases), (C) July 1995 through June 1996 (188 cases), (D) July 1996 through November 1998 (72 cases); chloropleth (natural breaks of Jenks) with an increased BSE relative risk with darkness; the medium grey includes the relative risk equal to one.

detected after June 2001 was representative of BSE in that cohort, regardless of the date of diagnosis. The only impact of this potential bias and assumption on the disease mapping was an underestimation of the number of BSE cases and a corresponding loss of power. In spite of this, significant results were obtained even for the oldest cohorts in the Bayesian analysis. The consequence for the EMM test was that fewer BSE cases would be diagnosed in either the early or late birth cohorts, as was the case. A retrospective study of BSE cases diagnosed prior to 2001 and prospective analysis of BSE cases diagnosed since 2004 could help quantify the impact of the potential detection bias.

Very few (29) BAB cases detected from July 2001 through December 2003 were born before July 1993. Given this we categorised the date of birth in trimesters for the
EMM test, we evaluated only cases born thereafter because there were only sparse data for the initial time period. These cases were kept for the mapping technique but were pooled with those born from July 1993 through June 1994 to provide an adequate number of point events (BSE cases) in order to produce coherent and interpretable maps. This subgroup represented $30 \%$ of the cases of the birth period January 1991 through June 1994. The map for this birth period (Fig. 3) shows a very focused risk in Brittany (Fig. 3), which was the putative starting point of the epidemic in France.

As shown in the results, the EMM test was influenced by the annual and seasonal infection. The latter might be influenced by seasonal variability in birthing, feeding practices or other BSE-exposure risks of young animals. The significant results of the space and time clustering of the BSE cases could have been the consequence of variations in the age structure as well as in the distribution of season of birth of the population at risk in different regions. We verified that apart from the difference between dairy and beef cattle, which was already taken into account in the study, these parameters did not vary between regions. BASB cases were not numerous enough to analyse dairy and beef cattle separately, which could have obscured peaks occurring in either production type. It should also clearly be stated, that, since the population at risk was not taken into account in the EMM test and because all cases from the birth cohorts could not be detected (see previous discussion), the incidence and therefore the infection pressure might be higher in other time periods and regions than the ones with a peak of cases. Concerning the disease mapping, the method used compared the risk in each hexagon to the average risk for each period of birth, independently from the others. The chloropleth mapping shows regions that had statistically significantly higher risk during that birth period. While SIR cannot be compared between maps, contrasts of the BSE risk between maps were comparable and we based the discussion on them. 
The EMM test identified regions in the southwest of France with a specific peak period for the birth of the BAB cases, from October through December 1995 (fall); it was the same for dairy (Poitou-Charentes, Aquitaine) and beef cattle (Midi-Pyrénées). This is particularly surprising because although one-third of the dairy calvings occur in the fall (data from French BSE surveillance), less than $20 \%$ of beef cattle were born in the fall. This peak was different from those of dairy cattle elsewhere (JulySeptember 1995) and beef cattle elsewhere (January-March 1995). Concerning the BASB cases, a significant peak was observed with the EMM test in the Pays-dela-Loire region, for those animals born in the "July-September 1997" period. A peak was already observed in this region for the $\mathrm{BAB}$ cases born in the same trimester, two years earlier. The results of the disease mapping support these findings and give a complementary point of view on the evolution of the BSE risk. The peak of dairy and beef cases born in the fall 1995 in the southwest of France is in agreement with the findings of an increased risk in the southwest for cattle born from July 1995 through June 1996 (Map C on Fig. 3). In the same manner, the peak of BASB cases in the Pays-de-la-Loire region matched the higher risk observed in the west of France for animals born after June 1996 (Map D on Fig. 3).

Each of the four maps built according to successive birth periods of cases detected between 2001 and 2003 showed a spatially significant heterogeneity of the risk and different areas at highest risk from one birth cohort to another. Few hexagons had a significantly higher relative risk (Fig. 4), and their locations matched the areas highlighted with the darkest colours of the chloropleth in the maps (Fig. 3). Comparing the contrasts of the SIR in the successive maps highlights how the areas with the highest risk changed depending on birth cohorts. It has to be noted that a change in the colour for a given area means that its relative BSE risk (rank) has changed between periods, but not necessarily its absolute risk. More

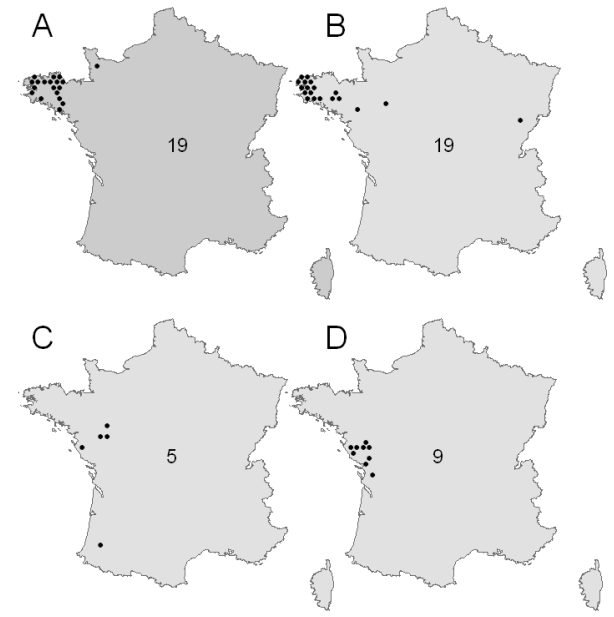

Figure 4. Hexagons with an SIR above the 99.5\% quantile of the prediction interval for the four different periods of birth: (A) January 1991 through June 1994, (B) July 1994 through June 1995, (C) July 1995 through June 1996, (D) July 1996 through November 1998.

precisely, the risk was significantly higher in some hexagons located in the Brittany region for the cohorts born until June 1994. This is the area where the first BSE case was detected in France in 1991 [16], and most of the BAB cases discovered before 1999. In the following birth cohort, born from July 1994 through June 1995, various French regions including the centre and the east showed an increased risk for BSE, the highest risk still being located in Brittany. In the following birth cohort, born from July 1995 through June 1996, the highest BSE risk moved to the southwest of France and the Pays-de-la-Loire region. Brittany was not the area with the highest BSE risk in a birth cohort anymore. Finally, for the BASB cases, born from July 1996 through October 1998 , the risk of BSE was the highest in the Pays-de-la-Loire region, located in southern Brittany.

Different factors might explain the temporal evolution and the spatial distribution of the areas at the highest risk of BSE. 
Firstly, as observed in the United Kingdom [29], it is probable that from the start of the BSE epidemic in France - not defined because the situation is unknown for cohorts born before 1991 - the spread of BSE might have resulted from the recycling of the BSE agent through the rendering process. The ban against MBM for cattle was already in force at that time but it is assumed that infection still continued because of cross-contaminations of MBM containing BSE infectivity from monogastric to ruminant feed [1]. There is no clear explanation why the southwest of France became at higher risk later, for the following birth cohort (July 1995-June 1996). The EMM test showed that this was the case for both dairy and beef cattle in contiguous regions, and more specifically, the peak was for dairy and beef cattle born in the fall of 1995 despite the fact that most beef cows were born in the winter or spring. It can be hypothesised that an unknown event might have occurred in the fall of 1995 resulting in an elevated risk of BSE infection in this region.

Secondly, control efficiency likely varied between regions. As observed from the abattoir and fallen stock surveillance data, before the strong decrease of the risk that started on the birth cohort 1995-1996, the BSE prevalence was still increasing significantly in the overall French territory between the birth cohorts July 1993 through June 1994 and July 1994 through June 1995 [19]. However, the prevalence was similar for both birth cohorts in western France (3 regions including Brittany) [24]. It has been hypothesised [19] that exposure decreased earlier in western France than in any other part of France because feed manufacturers were more aware of the disease in this region. This is likely because the BSE index cases were in western France and the apparent incidence was higher than elsewhere. It follows that more stringent BSE transmission control measures, including separation between ruminant and monogastric feed chains, were probably taken earlier than that required by law in this region. The fact that the relative risk for cases born between July 1995 and June 1996 (Map C on Fig. 3) varied substantially between neighbouring hexagons in western France might be due to the fact that more stringent measures were not taken uniformly in the area.

One of the main interests of this study was to compare the spatial distribution of the BASB and BAB cases. As already mentioned in Abrial et al. [2], the risk of BSE for the French BASB cases was not randomly distributed, which does not support a "spontaneous" or "sporadic" form of BSE. Despite the fact that the risk became more uniform for the BARB cases in the UK as compared to the BAB, Wilesmith et al. ${ }^{3}$ and DEFRA $^{4}$ also found a spatial cluster for the BARB cases. British BARB cases cannot be compared fully to French BASB; however, the epidemiological findings on them are meaningful for our study since they were born when a comprehensive feed ban was in force. Wilesmith et al. ${ }^{3}$ and DEFRA ${ }^{4}$ found a difference in BSE risk between dairy and beef cattle for the BARB cases, as seen previously for the $\mathrm{BAB}$, which is not consistent with a genetically based origin since all breeds appear to be susceptible to BSE. They observed the occurrence of two pairs - detected on the same farm - of cases in their small case series, which is a strong argument against these cases being spontaneous. In our study, it follows from the comparison of the contrasts of the SIR in the disease mapping of the different birth periods (Figs. 3 and 4) that the spatial distribution of the risk for the BASB cases in France, born after June 1996, matched the spatial pattern of risk in the preceding $\mathrm{BAB}$ birth cohort. The areas with the highest risk for the birth cohort preceding those of the BASB included the southwest as well as the Pays-de-la-Loire region, which had the highest incidence of BASB. The origin of

4 DEFRA 2003, Transmissible Spongiform Encephalopathies (TSEs) in Great Britain, 2003 - A progress report, 51p., http://www.defra.gov.uk/ animalh/bse/publications/progress/dec03/order.pdf [consulted 10 March 2005]. 
infection for the BASB has the same global spatial distribution as that of the $\mathrm{BAB}$, suggesting it might be the same.

Both environmental and maternal transmission might also lead to similar spatial distributions of BAB and BASB risk. However, there are two strong arguments against these hypotheses. First, the infection resulting from environmental or maternal transmission would be delayed, because it would require a latent and preclinical period likely exceeding several years. Second, the area with the oldest and highest BSE risk, Brittany, became a relatively low BSE-risk area for cohorts born after June 1995. These results show that the risk of experiencing BASB cases in an area was not linked to a higher past incidence of BSE in this area. This does not prove that an environmental or maternal infection did not occur, but it strongly supports the fact that the environmental or maternal transmission was not the main origin of the BASB cases. Wilesmith et al. ([32] and report, $2003^{3}$ ) reached the same conclusion for the BARB cases. Very few of them occurred in herds previously affected by BSE, and most dams of BARB cases were not affected by BSE 6 months and more after the birth of the case. In their report $^{3}$ (2003), Wilesmith et al. mentioned two additional possible sources that they want to address, the use of abattoir waste and sewage sludge. The preliminary results [18] from case studies carried out on farms affected with BASB cases did not report such practices in France.

From our findings, it appears that the spatial pattern of BASB BSE risk resembled the spatial pattern observed for the preceding $\mathrm{BAB}$ cases in France. Given our assumptions concerning the origin of the BAB cases [1], it is likely foodborne. This statement requires further scientific input that might be provided by a case-control study, as well as more in-depth investigation on BASB cases. It has to be emphasised that this study shows no indication for a spontaneous or environmental infection of the majority of the BASB cases as well as for the BAB cases. However, we cannot ignore the fact that these infection sources may exist at low levels.

\section{ACKNOWLEDGEMENTS}

The authors thank Éric Morignat (Agence Française de Sécurité Sanitaire des Aliments) and Daniel Lafon (Brigade Nationale d'Enquêtes Vétérinaires et Phytosanitaires Ministry of Agriculture) who provided the data of the surveillance programme of BSE. The study was funded in the frame of the "Groupement d'Intérêt Scientifique Infections à prions".

\section{REFERENCES}

[1] Abrial D., Calavas D., Jarrige N., Ducrot C., Poultry, pig and the risk of BSE following the feed ban in France - Ecological analysis, Vet. Res. 36 (2005) 615-622.

[2] Abrial D., Calavas D., Jarrige N., Ducrot C., Spatial heterogeneity of the risk of BSE in France following the ban of meat and bone meal in cattle feed, Prev. Vet. Med. 67 (2005) 69-82.

[3] AFSSA, Les risques sanitaires liés aux différents usages des farines et graisses d'origine animale et aux conditions de leur traitement et de leur élimination, AFSSA Ed., MaisonsAlfort, 2001, $200 \mathrm{p}$.

[4] Besag J, York J., Mollié A., Bayesian image restoration, with two applications in spatial statistics, Annals of the Institute of Statistical Mathematics 43 (1991) 1-59.

[5] Browne W.J., Applying MCMC methods to multilevel models, Ph.D. thesis in statistics, University of Bath, 1998, $211 \mathrm{p}$.

[6] Calavas D, Ducrot C., Baron T., Morignat E. Vinard J.-L., Biacabe A.G., Madec J.-Y., Bencsik A., Debeer S., Eliazsewicz M., Prevalence of BSE in western France by screening cattle at risk: preliminary results of a pilot study, Vet. Rec. 149 (2001) 55-56.

[7] Calavas D., Morignat E., Cazeau G., Ducrot C., ESB: une synthèse de l'épidémiologie en France, Bulletin des Groupements Techniques Vétérinaires 95 (2003) 23-30.

[8] Carpenter T.E., Methods to investigate spatial and temporal clustering in veterinary epidemiology, Prev. Vet. Med. 48 (2001) 303-320. 
[9] Cazeau G., Ducrot C., Morignat E., Lauvergne N., Philippe S., Cohen C., Calavas D., Surveillance clinique de l'ESB en France, Étude quantitative des caractéristiques des animaux et des signes cliniques observés en cas de suspicion, Rev. Med. Vet. 153 (2002) 785794.

[10] Clayton D., Kaldor J., Empirical bayes estimates of age-standardized relative risks for use in disease mapping, Biometrics 43 (1987) 671-681.

[11] Cuenot M., Calavas D., Abrial D., Gasqui P., Cazeau G., Ducrot C., Temporal and spatial patterns of the clinical surveillance of BSE in France analysed from January 1991 to May 2002 through a vigilance index, Vet. Res. 34 (2003) 261-272.

[12] Donnelly C.A., Likely size of the French BSE epidemic, Nature 408 (2000) 787-788.

[13] Ducrot C., Roy P., Morignat E., Baron T., Calavas D., How the surveillance system may bias the results of analytical epidemiological studies on BSE: prevalence among dairy versus beef suckler cattle breeds in France, Vet. Res. 34 (2003) 185-192.

[14] Ederer F., Myers M.H., Mantel N., A statistical problem in space and time: do leukemia cases come in clusters? Biometrics 20 (1964) 626-638.

[15] Feller W., An introduction to probability theory and its applications, 2nd ed., Wiley, New York, 1957.

[16] Gouello L., Analyse épidémiologique du premier cas d'encéphalopathie spongiforme bovine en France, Épidémiologie et Santé Animale 19 (1991) 63-70.

[17] Heidelberger P., Welch P.D., Simulation run length control in the presence of an initial transient, Oper. Res. 31 (1983) 1109-1144.

[18] Jarrige N., Ducrot C., Lafon D., Thiebot B., Calavas D., Potential sources of infection for the French BSE cases born since 1996, Vet. Rec. (2005) in press.

[19] Labonnardière C., Calavas D., Abrial D., Morignat E., Ducrot C., Estimating the trend of the French BSE epidemic over six birth cohorts through the analysis of abattoir screening in 2001 and 2002, Vet. Res. 35 (2004) 299-308.

[20] Mantel N., Kryscio R.J., Meyers M.H., Tables and formulas for extended use of the EdererMeyers-Mantel disease clustering procedure, Am. J. Epidemiol. 104 (1976) 576-584.
[21] Mollié A., Bayesian and Empirical Bayes approaches to disease mapping, in: Lawson A., Biggeri A., Böhning D., Lesaffre E., Viel J.-F., Bertollini R. (Eds.), Disease mapping and risk assessment for public health, Wiley, Chichester, 1999, pp. 15-29.

[22] Mollié A., Bayesian mapping of Hodgkin's disease in France, in: Elliot P., Wakefield J., Best N., Briggs D. (Eds.), Spatial epidemiology, Oxford University, Oxford, 2000, pp. 266-285.

[23] Morignat E., Ducrot C., Roy P., Baron T., Vinard J.-L., Biacabe A.G., Madec J.-Y., Bencsik A., Debeer S., Eliazsewicz M., Calavas D., Targeted surveillance to assess the prevalence of BSE in high-risk populations in western France and the associated risk factors, Vet. Rec. 151 (2002) 73-77.

[24] Morignat E., Ducrot C., Roy P., Cohen C.H., Calavas D., Prevalence of BSE in cattle found dead, euthanased or emergency slaughtered on farms in western France in 2000, 2001 and 2002, Vet. Rec. 155 (2004) 481-485.

[25] Moynagh J., Schimmel H., Tests for BSE evaluated, Nature 400 (1999) 105.

[26] Spiegelhalter C., Thomas A., Best N., Gilks W., BUGS: Bayesian Inference Using Gibbs Sampling, Cambridge, MRC Biostatistics Unit, 2000 .

[27] Stark C.R., Mantel N., Lack of seasonal- or temporal-spatial clustering of Down's syndrome births in Michigan, Am. J. Epidemiol. 86 (1967) 199-213.

[28] Stark C.R., Mantel N., Temporal-spatial distribution of birth dates for Michigan children with leukemia, Cancer Res. 27 (1967) 17491775.

[29] Stevenson M.A., Morris R.S., Pfeiffer D.U., Lin D., Wilesmith J.W., Ryan J.B.M., Lawson A.B., Descriptive spatial analysis of the epidemic of bovine spongiform encephalopathy in Great Britain to June 1997, Vet. Rec. 147 (2000) 379-384.

[30] Supervie V., Costagliola D., The unrecognised French BSE epidemic, Vet. Res. 35 (2004) 349-362.

[31] Wilesmith J.W., Wells G.A.H., Cranwell M.P., Ryan J.B.M., Bovine spongiform encephalopathy: epidemiological studies, Vet. Rec. 123 (1988) 638-644.

[32] Wilesmith J.W., Preliminary epidemiological analyses of the first 16 cases of BSE born after July 311996 in Great Britain, Vet. Rec. 151 (2002) 451-452. 